\title{
Trois sépultures atypiques du Second âge du Fer à Reviers (Calvados)
}

Three atypical Iron Age graves from Reviers (Calvados)

Sophie Oudry-Braillon et Cyrille Billard

\section{(2) OpenEdition}

\section{Journals}

Édition électronique

URL : https://journals.openedition.org/rao/830

DOI : $10.4000 /$ rao. 830

ISBN : 978-2-7535-1609-0

ISSN : $1775-3732$

\section{Éditeur}

Presses universitaires de Rennes

\section{Édition imprimée}

Date de publication : 31 décembre 2009

Pagination : 105-115

ISBN : 978-2-7535-1086-9

ISSN : 0767-709X

\section{Référence électronique}

Sophie Oudry-Braillon et Cyrille Billard, «Trois sépultures atypiques du Second âge du Fer à Reviers (Calvados) », Revue archéologique de l'Ouest [En ligne], 26 | 2009, mis en ligne le 31 décembre 2011, consulté le 17 août 2022. URL : http://journals.openedition.org/rao/830 ; DOI : https://doi.org/ $10.4000 /$ rao.830 


\title{
Trois sépultures atypiques du Second âge du Fer à Reviers (Calvados)
}

\author{
Three atypical Iron Age graves from Reviers (Calvados)
}

\author{
Sophie Oudry-Braillon * et Cyrille Billard **
}

\begin{abstract}
Résumé : Trois sépultures du Second âge du Fer, mises au jour en 2000 à Reviers (Calvados), montrent un mode d'inhumation inhabituel. Chacun des individus, adulte, a été enterré assis dans une fosse circulaire, avec les membres fléchis et le haut du corps penché vers l'avant. Après une étude précise des positions d'inhumation et des propositions de restitution de l'espace de décomposition des cadavres, nous établissons une comparaison avec des sites cultuels ayant livré des os humains erratiques ou non et les individus inhumés en position assise. Au terme de cette étude, l'interprétation de ce type de sépulture pose toujours problème.

Abstract: Three Second Iron Age burials, found in 2000 in Reviers (Calvados - France), show an uncommon type of inhumation. Every individual, of an adult age, was buried sitting in a circular pit, limbs bent and the upper half of the body leaning forward. After studying the position of each individual and proposing a reproduction of the decomposition space of the corpses, we compare these burials with worshiping sites yielding human remains and sitting individuals. This comparison done, we find that the interpretation of this type of burial remains problematic.
\end{abstract}

Mots clés : inhumation, position assise, Second âge du Fer, culte, religion.

Key words: burial, sitting position, Second Iron Age, worship, religion.

\section{INTRODUCTION}

La commune de Reviers est située dans le Calvados, au nord-ouest de la Plaine de Caen. Le site du Champ de Bény - parcelle ZL 4 - est localisé dans la partie est de la commune actuelle, sur le rebord d'un plateau crayeux dominant un méandre de la vallée de la Seulles, fleuve qui se jette dans la mer à environ $5 \mathrm{~km}$ au nord (fig. 1 et 2). Le terrain naturel, en nette pente en direction de la vallée, est composé de calcaire en plaquettes fortement altérées et localement mêlées à une matrice argilo-limoneuse.

Le site a été découvert lors d'une campagne de sondages réalisés par le Service régional de l'Archéologie de BasseNormandie en février 2000, sur une surface de plus d'un hectare (fig. 2). Ces sondages, préalables à un projet de lotissement communal, ont été motivés par la présence proche d'un important cimetière du haut Moyen Âge, connu depuis le XIx ${ }^{e}$ siècle et partiellement fouillé par Arcisse de Caumont en 1836. Un diagnostic archéologique a d'ailleurs été réalisé sur ce cimetière en novembre 2000, afin d'en préciser l'étendue et la densité des sépultures.

Les sondages de février 2000 n'ont pas mis au jour l'extension escomptée du cimetière du haut Moyen Âge, mais trois sépultures individuelles datées de la période protohistorique ainsi que deux fosses. Une surface d'environ $2000 \mathrm{~m}^{2}$ a été décapée autour des fosses sépulcrales pour analyser leur contexte. Les sépultures étant les seuls éléments positifs de cette opération, elles ont été fouillées à la suite du décapage,

* INRAP Nord-Pas-de-Calais - UMR 7041 - ZI La Pilaterie, 11 rue des Champs, 59650 Villeneuve-d'Ascq.

** DRAC de Basse-Normandie, Service régional de l'Archéologie - 13bis rue Saint-Ouen 14052 Caen cedex 04. 

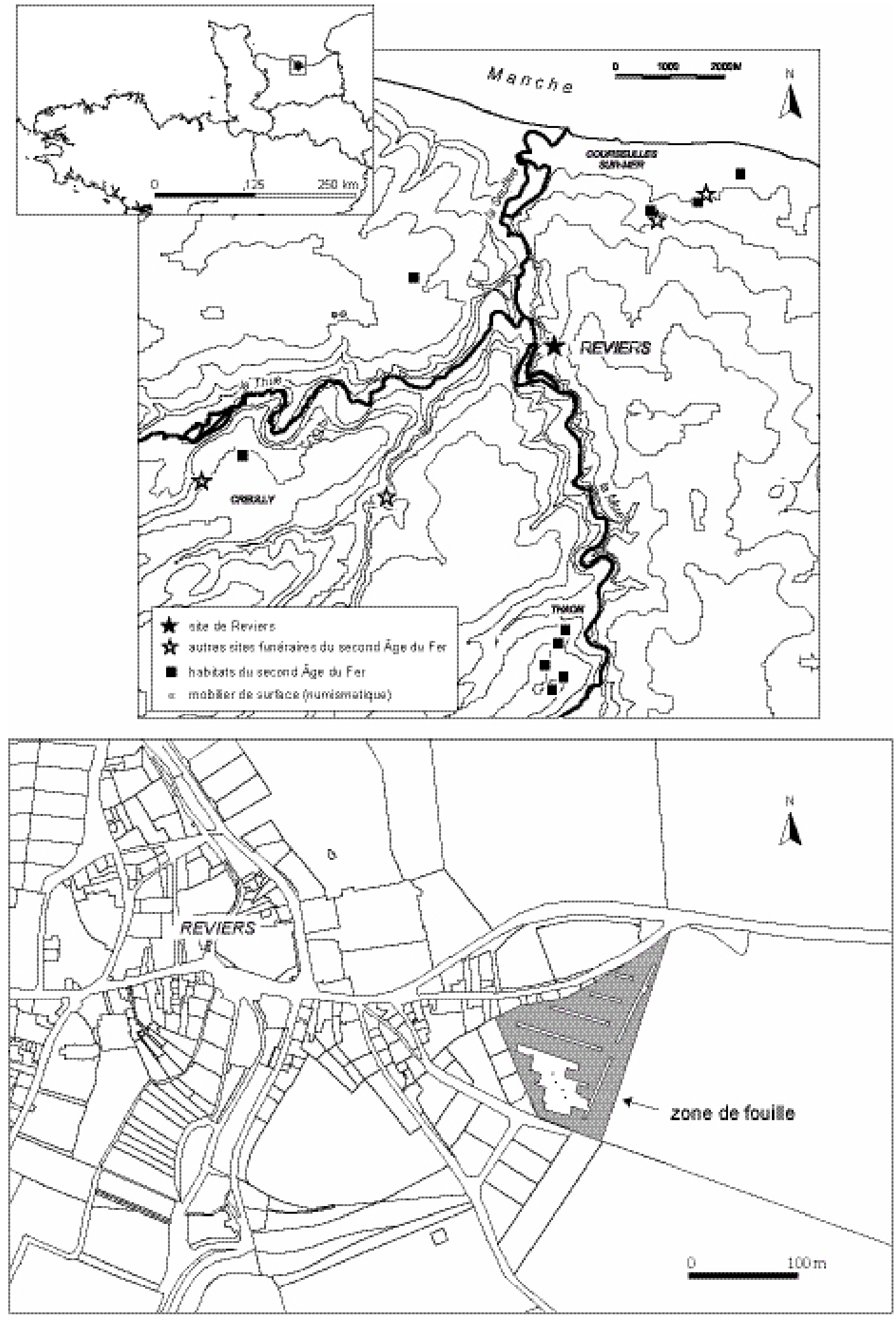

Figure 1 : Le site de Reviers et son environnement archéologique au second Âge du Fer (équidistance des courbes de niveau : $10 \mathrm{~m}$ )(cartographie Anne Ropars, SRA de Basse-Normandie - cellule carte archéologique). Figure 1: Reviers and its archaeological surroundings during the Second Iron Age. Equidistance of the contour lines: $10 \mathrm{~m}$. 


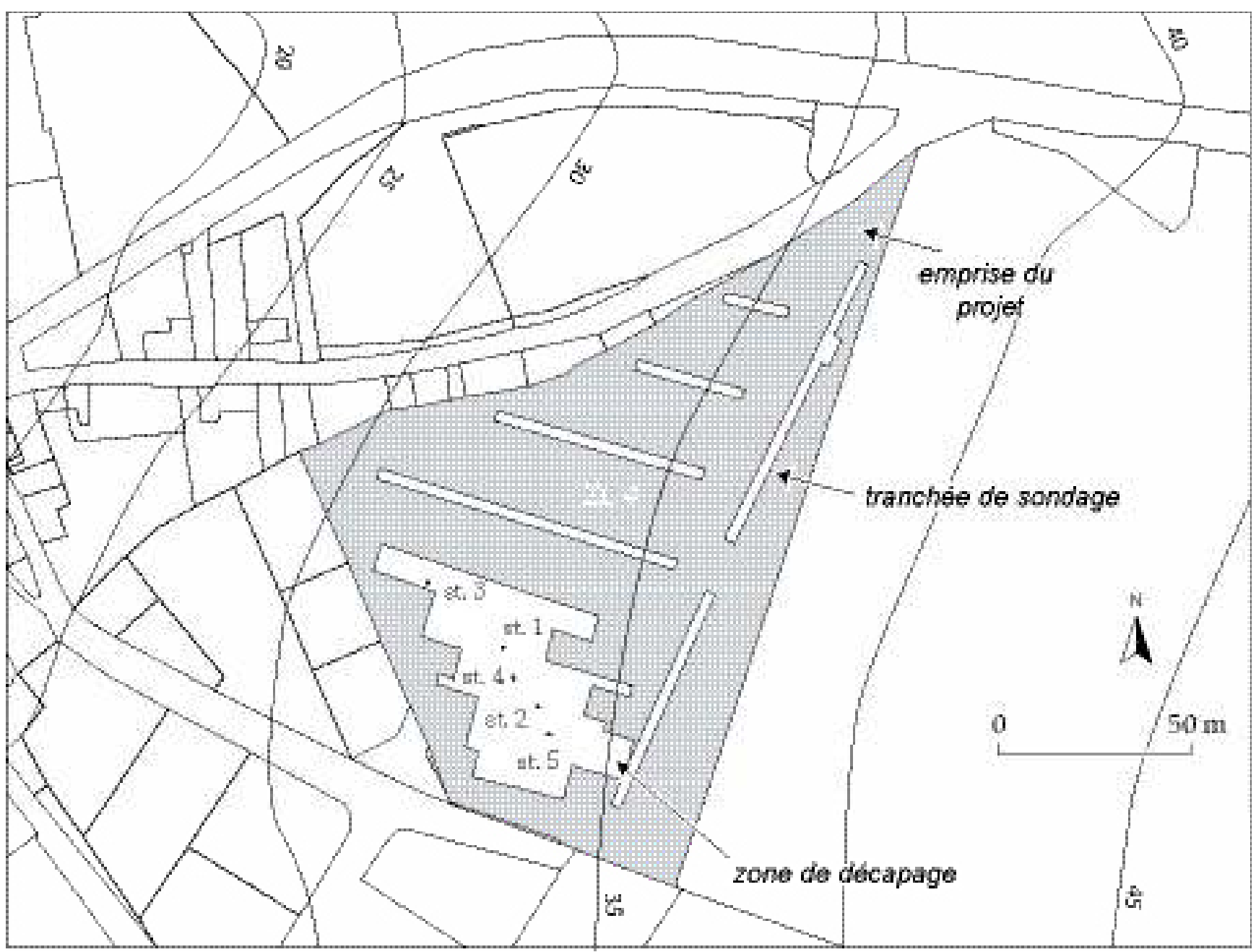

Figure 2 : Reviers, localisation des structures étudiées.

Figure 2: Site plan.

d'autant que la position curieuse des inhumés et la localisation du site au cœur de la zone du débarquement de 1944 soulevaient des doutes quant à leur datation. Chacun des individus a fait l'objet d'un démontage précis sur plusieurs niveaux avec enregistrement de la position et de l'altitude des ossements.

\section{LES TROIS SÉPULTURES ASSISES}

\section{Contexte et environnement archéologique}

Les trois sépultures (st. 1, 2 et 5) sont grossièrement alignées selon un axe nord-nord-ouest/sud-sud-est et sont distantes au maximum de $25 \mathrm{~m}$. Hormis deux autres fosses (st. 3 et 4), qui n'ont livré aucun matériel, on ne connait rien de l'environnement immédiat du site, les autres tranchées de sondage s'étant révélées négatives. La cartographie des sites du Second Âge du Fer à Reviers et dans les communes environnantes ne met pas en évidence d'occupations particulières à proximité, tels qu'oppidum, nécropole ou édifice cultuel. Cette zone est toutefois densément occupée aux époques protohistoriques, comme en témoignent les données des prospections menées sous la direction de Guy San Juan (Desloges et al., 1999).

\section{Descriptif général des fosses}

Leurs caractéristiques principales sont synthétisées dans le tableau 1. Les excavations retrouvées sont peu profondes en raison d'une érosion importante du site, due à sa position en hauteur sur une légère pente. Les trois fosses sépulcrales étant grossièrement circulaires, l'orientation retenue a été celle de l'individu : l'individu de la st. 1 fait face à l'ouest; 
celui de la st. 2 fait face à l'ouest sud-ouest et celui de la st. 5 fait face à l'ouest, mais la partie supérieure de son corps est penchée vers la gauche et fait donc face à l'ouest-sud-ouest. Tous les individus sont placés dos à la pente et face orientée vers le fond de la vallée.

\section{Mobilier et datation}

Les seuls éléments de mobilier disponibles proviennent de la st. 4 (qui ne renferme pas de squelette); il s'agit de quelques petits tessons protohistoriques, sans qu'il soit possible de préciser. Une datation radiocarbone a donc été réalisée sur les ossements de l'individu de la st. 5 ; la fourchette obtenue est de 349 à 2 avant J.-C. cal. (Lyon-1786 - GrA 20813 : $2110+/-45 \mathrm{BP})$. Une dent d'herbivore a été découverte dans la st. 2 et une canine de petit carnivore dans la st. 5 mais il est difficile de savoir si la présence de ces deux dents est intentionnelle ou si elles étaient déjà présentes dans ce qui deviendra le remplissage de la fosse. Dans le cas de la st. 5, la dent a été découverte parmi les os de la main gauche.

\section{La position des corps}

\section{La sépulture 1}

La plus grande partie des ossements conservés appartient à la partie inférieure du corps (fig. 3); en effet, l'érosion importante de cette fosse (profondeur conservée $20 \mathrm{~cm}$ ) a détruit une part importante du squelette. Le pendage du fond de la fosse fait qu'elle est plus profonde du côté du bassin. L'individu est assis, le membre inférieur gauche très fléchi, genou devant le corps, pied ramené vers le bassin : l'intérieur de la cheville est posé sur le fond de la fosse. Le

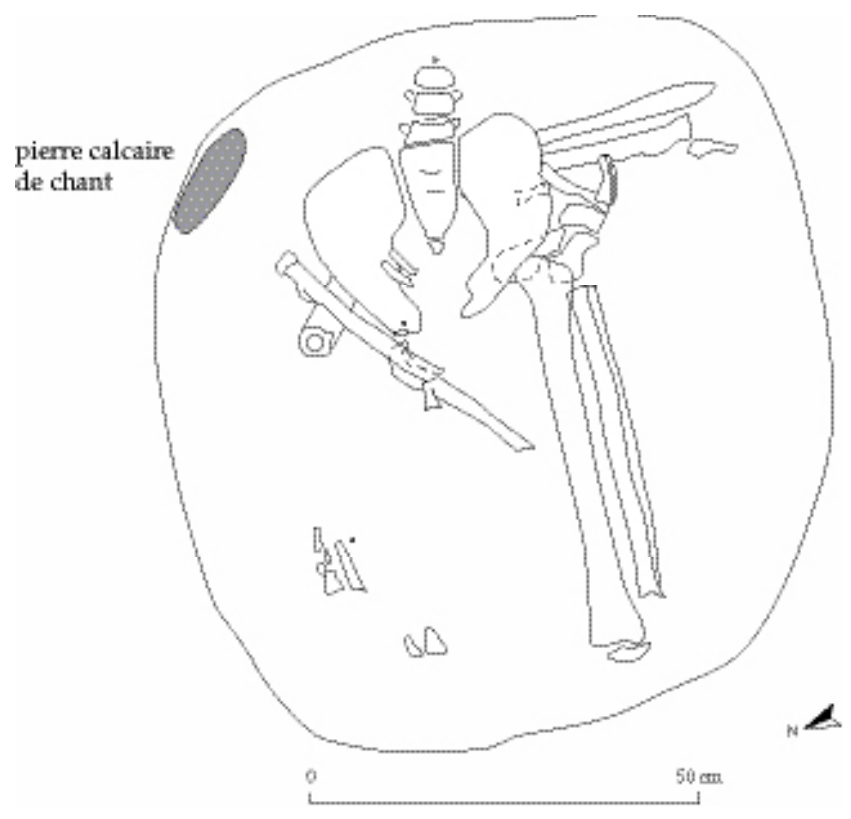

Figure 3 : Reviers, plan de la sépulture 1. Figure 3: Plan of Burial 1.

reste du fémur droit (le tiers proximal) semble indiquer que le membre inférieur droit était fléchi avec le genou devant le corps, un peu décalé vers l'extérieur et en hauteur. Un fragment de talus repose sur le fond de la fosse à l'opposé du bassin. Ce dernier, en connexion, et le rachis conservé (deux dernières vertèbres lombaires) sont en appui contre la paroi de la fosse. Le membre supérieur gauche était vraisemblablement fléchi, mais il n'en reste que l'avant-bras, encore en connexion : il passe derrière le bassin, avec le coude plus

\begin{tabular}{|l|c|c|c|c|c|}
\hline St. 1 & $94 \times 86 \mathrm{~cm}$ & $20 \mathrm{~cm}$ & $\begin{array}{c}\text { Frofondeur par rapport } \\
\text { au toit du calcaire }\end{array}$ & $\begin{array}{c}\text { Rub-circulaire, parois } \\
\text { verticales, fond plat } \\
\text { avec un pendage vers le } \\
\text { sud-est }\end{array}$ & $\begin{array}{c}\text { Limon argileux brun orangé, riche en } \\
\text { plaquettes calcaires }\end{array}$ \\
\hline St. 2 & $90 \times 90 \mathrm{~cm}$ & $33 \mathrm{~cm}$ & $\begin{array}{c}\text { Circulaire, parois sub- } \\
\text { verticales, fond plat et } \\
\text { horizontal }\end{array}$ & $\begin{array}{c}\text { Limon argileux brun orangé, riche en blocs } \\
\text { calcaires }\end{array}$ \\
\hline St. 3 & $90 \times 100 \mathrm{~cm}$ & $15 \mathrm{~cm}$ & Sub-circulaire & $\begin{array}{c}\text { Limon argileux brun orangé, riche en } \\
\text { plaquettes calcaires }\end{array}$ & oui \\
\hline St. 4 & $100 \times 135 \mathrm{~cm}$ & $30 \mathrm{~cm}$ & Ovale & $\begin{array}{c}\text { Remplissage identique aux autres, mais } \\
\text { composante limoneuse plus marquée }\end{array}$ & non \\
\hline St. 5 & $100 \times 90 \mathrm{~cm}$ & $40 \mathrm{à} 42 \mathrm{~cm}$ & $\begin{array}{c}\text { Sub-circulaire, parois } \\
\text { sub-verticales, fond } \\
\text { grossièrement horizontal }\end{array}$ & $\begin{array}{c}\text { Limon argileux brun orangé, riche en } \\
\text { plaquettes calcaires }\end{array}$ \\
\hline
\end{tabular}

Tableau 1 : Récapitilatif des caractéristiques des cinq fosses de Reviers.

Table 1: Main characteristics of the five Reviers pits. 
élevé que le poignet. L'avant-bras droit est localisé devant le bassin, avec le coude vers l'extérieur du corps et le poignet (non conservé) vraisemblablement situé entre les deux membres inférieurs, vers le centre de la fosse; le radius et l'ulna sont déconnectés l'un de l'autre : l'ulna a glissé le long du radius vers le centre de la fosse. Ce qui reste des phalanges des mains ne permet pas de restituer leur position. Des fragments de côtes sont dans le volume du bassin et un fragment de la clavicule droite est resté en hauteur au-dessus du pied gauche. Enfin, une plaquette calcaire était posée de chant le long de la paroi est de la fosse.

\section{La sépulture 2}

L'individu est également assis, mieux conservé que dans la sépulture précédente (fig. 4); il manque cependant le crâne, la première vertèbre cervicale, la partie proximale des deux humérus et la partie supérieure de la scapula gauche. Le membre inférieur gauche est très fléchi, avec le genou en hauteur et légèrement orienté en dehors; le pied est ramené contre le bassin, avec l'intérieur de la cheville dirigé vers le ciel; les phalanges du pied ne sont pas conservées. Le membre inférieur droit est légèrement fléchi, avec le genou en hauteur (patella en connexion) et penché vers l'extérieur; le pied est situé loin en avant du corps, partiellement contre la paroi de la fosse, le talon sur le fond; ce pied est fléchi et apparaît en vue supéro-médiale. Le

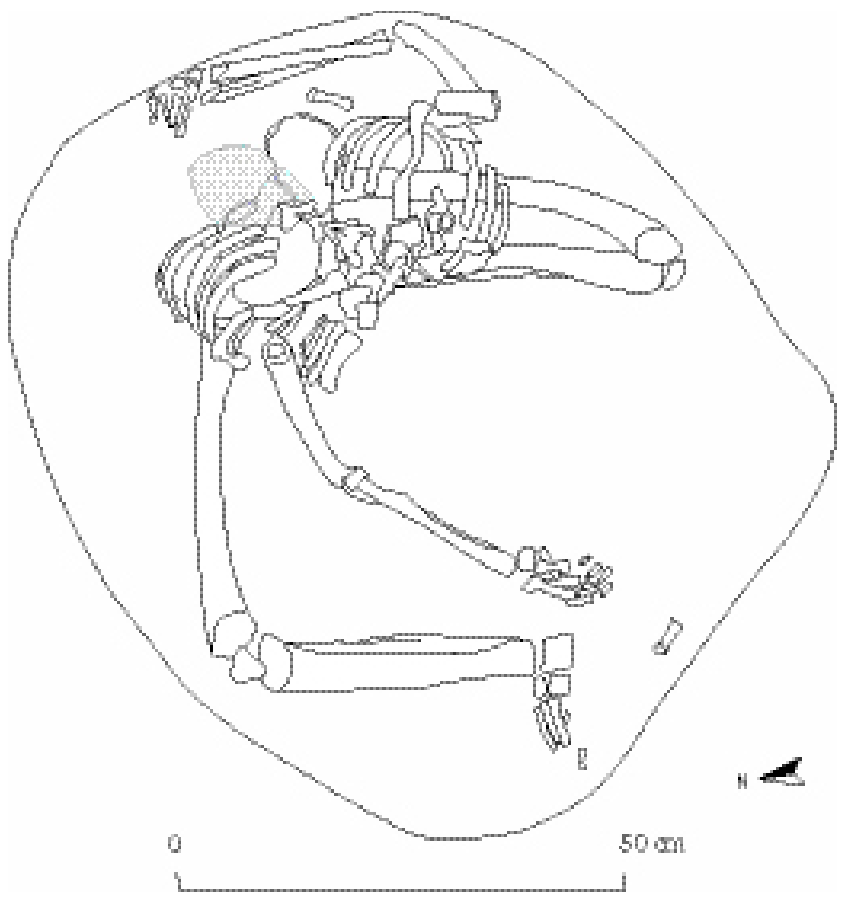

Figure 4 : Reviers, plan de la sépulture 2 (en grisé, le rachis mal conservé).

Figure 4: Plan of Burial 2 (ill-preserved backbone is shaded). bassin, contre la paroi de la fosse, est bien connecté et apparaît en vue supérieure. Le tronc est penché vers l'avant et légèrement vers la gauche. Le membre supérieur droit est légèrement fléchi en avant du corps, la main reposant sur le fond de la fosse. Le membre supérieur gauche, fléchi, passe derrière le corps; le coude est en appui contre la paroi et la main, en vue dorsale, est plus haute que le coude, en position contrainte.

\section{La sépulture 5}

L'individu est assis; il est le mieux conservé des trois (fig. 5). Le membre inférieur gauche est très fléchi, avec le pied ramené devant le bassin, l'intérieur de la cheville dirigé vers le ciel; le genou est à plat sur le fond de la fosse. Le membre inférieur droit est fléchi vers l'extérieur, avec le genou en hauteur, le talon en avant du corps reposant sur le fond de la fosse et l'extérieur de la cheville dirigé vers le sol. Le bassin est en appui contre la paroi de la fosse et le tronc penché vers l'avant et vers la gauche, au-dessus du fémur gauche. Le crâne et la mandibule, en connexion entre eux et avec le rachis, sont en vue postérieure et inclinés vers l'avant, dans le prolongement du tronc. Le membre supérieur gauche est fléchi avec le coude en appui contre la paroi et la main contre l'extérieur du fémur gauche. Le membre supérieur droit est également fléchi, avec le coude légèrement en arrière du corps et la main en extension, posée en partie sur la cheville gauche.

\section{L'espace de décomposition : restitution}

Dans aucune des trois sépultures nous n'avons d'effets de délimitation linéaire ou de paroi, ni d'indice de coffrage ou d'aménagements particuliers de la tombe. Plusieurs arguments plaident en faveur d'un espace colmaté, et ce pour les trois individus.

Dans le cas de la sépulture 1, le radius et l'ulna droits sont en position haute par rapport au fond de la fosse, de même qu'une phalange de la main droite; le fragment de fémur droit est en position quasiment verticale, sans soutien; à noter aussi le maintien de la clavicule droite en hauteur et la patella gauche de chant, en connexion avec le reste du membre inférieur; le pied gauche est encore en connexion.

Dans le cas de la sépulture 2, les côtes sont maintenues en hauteur et ont conservé une bonne connexion avec les vertèbres thoraciques. Le rachis est maintenu en position instable et en connexion, depuis les dernières vertèbres cervicales jusqu'aux lombaires. L'humérus droit est en position quasiment verticale et le coude est en élévation sans soutien et en connexion stricte. Il n'y a pas d'ouverture du bassin; le radius droit est en équilibre instable sur l'ulna; les os de la main droite sont encore bien connectés entre eux. Les deux 
A

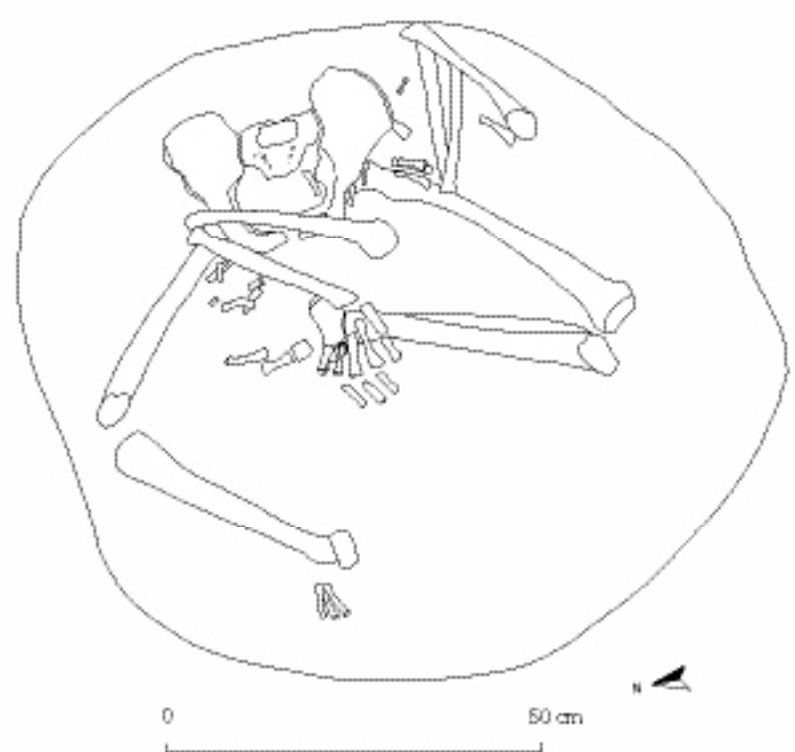

B

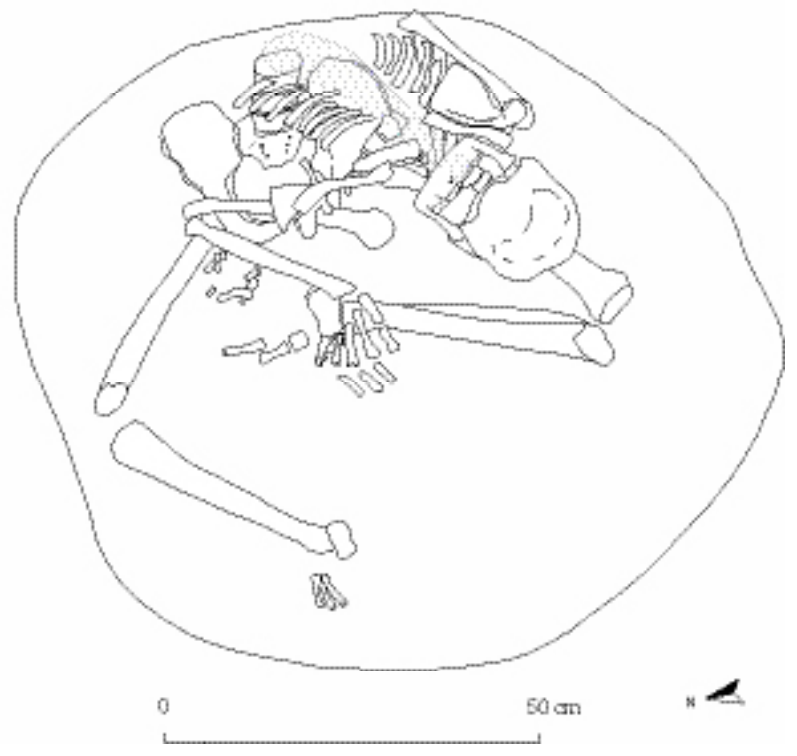

Figure 5 : Reviers, plans de la sépulture 5 (en grisé, le rachis mal conservé) : A, partie inférieure du corps (sans le rachis, le thorax et le crâne); B, ensemble du corps.

Figure 5: Plans of Burial 5 (ill-preserved backbone is shaded). A, inferior part of the corpse; $B$, complete corpse. patellas sont de chant et en connexion stricte avec le reste de leur membre inférieur respectif.

Dans le cas de la sépulture 5, enfin, le colmatage rapide de l'espace de décomposition est également l'hypothèse retenue en raison du maintien du crâne en position instable et de sa bonne connexion avec la mandibule, les dents et les cervicales. On peut aussi évoquer les épaules en élévation et la connexion en élévation de la clavicule, de l'humérus et de la scapula gauches.

D'une manière globale, rien ne permet de suggérer que la partie supérieure du corps (ou même seulement le crâne) ait pu rester visible hors de terre. Tout au plus peut-on affirmer, pour les deux sépultures les plus arasées, que le corps a été enterré à faible profondeur.

Il y a donc vraisemblablement un même type d'espace de décomposition pour les trois individus : un espace colmaté; cependant, plusieurs déplacements d'ossements limités au volume initial du corps ont été notés.

Dans le cas de l'individu 1, une incisive et trois fragments de côtes ont chuté dans le volume initialement occupé par le thorax. Le glissement de l'ulna droite le long du radius droit peut s'expliquer par la position de l'avant-bras juste sous le niveau de la terre végétale, ce qui aurait pu entraîner des déplacements ou des disparitions d'ossements, comme cela a vraisemblablement été le cas pour presque toute la partie supérieure et la moitié droite du corps. On peut aussi supposer que ce n'est pas l'ulna qui a chuté, mais le radius qui aurait été tiré vers le haut au moment de la disparition de l'humérus, entraîné par ce dernier.

Dans le cas de l'individu 2, l'axis est légèrement déconnecté; les deux premières côtes sont tombées dans le volume du thorax, de même que la clavicule droite et deux vertèbres cervicales, restées en connexion entre elles. La clavicule gauche plonge pour partie dans le volume du thorax (l'extrémité médiale est à $-17 \mathrm{~cm}$ et l'extrémité distale à $-8 \mathrm{~cm}$ ). Le manubrium a chuté sur le tibia gauche et on note une déconnexion peu importante entre le tarse proximal (talus et calcanéus en vue médio-supérieure) et le reste du tarse suivi des métatarsiens et des phalanges (en vue médiale).

Dans le cas de la structure 5, la base de la scapula droite est quasiment descendue entre les coxaux et le sternum, complet, est déconnecté dans le volume du thorax; la main gauche a bougé dans le volume initial des fesses.

Si ces derniers déplacements peuvent s'expliquer par l'existence d'espace vides secondaires qui se créent au cours de la décomposition du corps - notamment toutes les chutes dans le volume du thorax - d'autres déplacements ne trouvent pas nécessairement d'explication.

Pour la structure 1, un métatarsien gauche a migré à $10 \mathrm{~cm}$ du pied et la clavicule droite se trouve en hauteur au-dessus du pied gauche. 
Pour la structure 2, on note la disparition totale des phalanges du pied gauche et le déplacement important d'une phalange de chaque main (plus de $10 \mathrm{~cm}$ d'écart avec la main). Une incohérence importante est visible dans les faces d'apparition au niveau du membre supérieur gauche : la main est contrainte dans le dos et elle apparaît en vue dorsale; l'humérus apparaît logiquement en vue supéro-médiale. En revanche, le radius est en vue antérieure légèrement latérale et l'ulna en vue médiale, alors qu'ils devraient tous les deux être en vue postéro-médiale. Il y a donc une rotation de plus de $90^{\circ}$ du radius et ce n'est pas le même sens de rotation qui s'applique au radius et à l'ulna : la rotation est visiblement plus prononcée dans le cas de l'ulna. Le maintien de cette position non-naturelle implique peut-être la présence d'une ligature du membre supérieur; il est cependant difficile de préciser à quel niveau ce lien aurait été fixé.

Dans le cas de la structure 5, d'importants déplacements sont visibles au niveau du pied gauche, avec notamment le déplacement et la "remontée " d'un métatarsien derrière le crâne (à $-5 \mathrm{~cm}$ au lieu de $-36 \mathrm{~cm}$ ). L'absence de la patella gauche est curieuse : le genou reposant sur le fond de la fosse, elle aurait dû être conservée.

Dans certains cas, le déplacement des petits os et notamment des phalanges pourrait s'expliquer par la présence d'animaux fouisseurs : la dent de petit carnivore trouvée au niveau de la main gauche de l'individu de la st. 5 en est peut-être le témoin.

\section{L'identité biologique des individus}

Les ossements des trois individus sont moyennement conservés; de ce fait un certain nombre de surfaces articulaires ne sont pas observables.

- Âge : Nous avons affaire à trois sujets adultes d'après leur morphologie ou d'après leur schéma dentaire.

- Sexe : L'individu de la st. 5 est de sexe masculin d'après les mesures de ses coxaux (Murail et al. 2005). Les coxaux des deux autres sont trop mal conservés pour une détermination sexuelle fiable.

- Métrique : Très peu d'éléments sont disponibles. Une seule estimation de la stature a pu être réalisée à partir du radius pour l'individu de la st. $2: 165,5 \mathrm{~cm} \pm 3,5 \mathrm{~cm}$ (Olivier 1960).

- Pathologie : chez l'individu de la st. 2, un phénomène arthrosique de stade 3 sur 4 a été observé sur trois vertèbres lombaires. Chez celui de la st. 5, le talus et le calcanéus gauches sont soudés. Le plateau des deux dernières vertèbres lombaires présente un phénomène arthrosique de stade 2 sur 4. Des caries ont été observées en face occlusale sur la troisième molaire inférieure gauche et sur les sillons latéraux des trois molaires inférieures droites et sur les première et troisième molaires inférieures gauches. Enfin des dépôts de tartre ont été observés en face linguale sur toutes les molaires inférieures.

\section{Pistes de RÉflexion}

Ces trois sépultures présentent indéniablement des traits communs et par cela nous amènent à penser qu'elles sont sensiblement contemporaines, mais ce n'est qu'un postulat en l'absence de datation précise pour deux des trois sépultures.

Les traits communs sont tout d'abord la forme et les dimensions des fosses : il s'agit dans les trois cas d'une fosse grossièrement circulaire, taillée dans le substrat calcaire et dont les diamètres oscillent entre 90 et $100 \mathrm{~cm}$. Les différences de profondeur observées - de 20 à $42 \mathrm{~cm}$ - ne sont vraisemblablement dues qu'à une différence d'érosion. Les parois sont verticales et le fond est plan. Il faut rappeler leur alignement sensiblement parallèle aux courbes de niveau, et la position commune des trois individus " regardant " vers la vallée. Le remplissage des fosses, un limon argileux brun orangé riche en plaquettes calcaires, est identique dans les trois cas. Enfin, la position d'inhumation tout à fait inhabituelle des trois individus est certainement le trait commun le plus frappant (fig. 6) : on retrouve dans les trois cas une position assise, avec le tronc incliné vers la gauche et vers l'avant, le membre inférieur droit le plus étendu possible compte tenu des dimensions de la fosse. Du côté gauche, les membres présentent de légères variantes de position, même s'ils sont à chaque fois fléchis : dans la st. 1, le membre inférieur est très fléchi avec le pied vers l'extérieur et le membre supérieur est fléchi derrière le dos; dans la st. 2, le membre inférieur est fléchi avec le talon contre le pubis et le membre supérieur est fléchi dans le dos; enfin, dans la st. 5, le membre inférieur est moins fléchi, talon devant le bassin, et le membre supérieur est fléchi près du côté gauche du bassin. La position du tronc, ou de ce qu'il en reste, est identique dans les trois cas; on peut donc supposer que le haut du corps était dans une position similaire à chaque fois, notamment en ce qui concerne le crâne, qui apparaît en vue postérieure dans le seul cas où il est conservé.

Dans les trois cas également, le même espace de décomposition est restitué d'après les phénomènes taphonomiques observés : un espace colmaté qui a permis la chute de quelques os dans le volume du thorax lors de la décomposition du corps. On ne peut donc pas expliquer certains déplacements hors du volume initial du corps observés par endroits, hormis par l'intervention d'animaux fouisseurs.

Dans le cas des st. 1 et 2, on pourrait évoquer l'hypothèse d'un prélèvement du crâne après le dépôt du corps dans la 

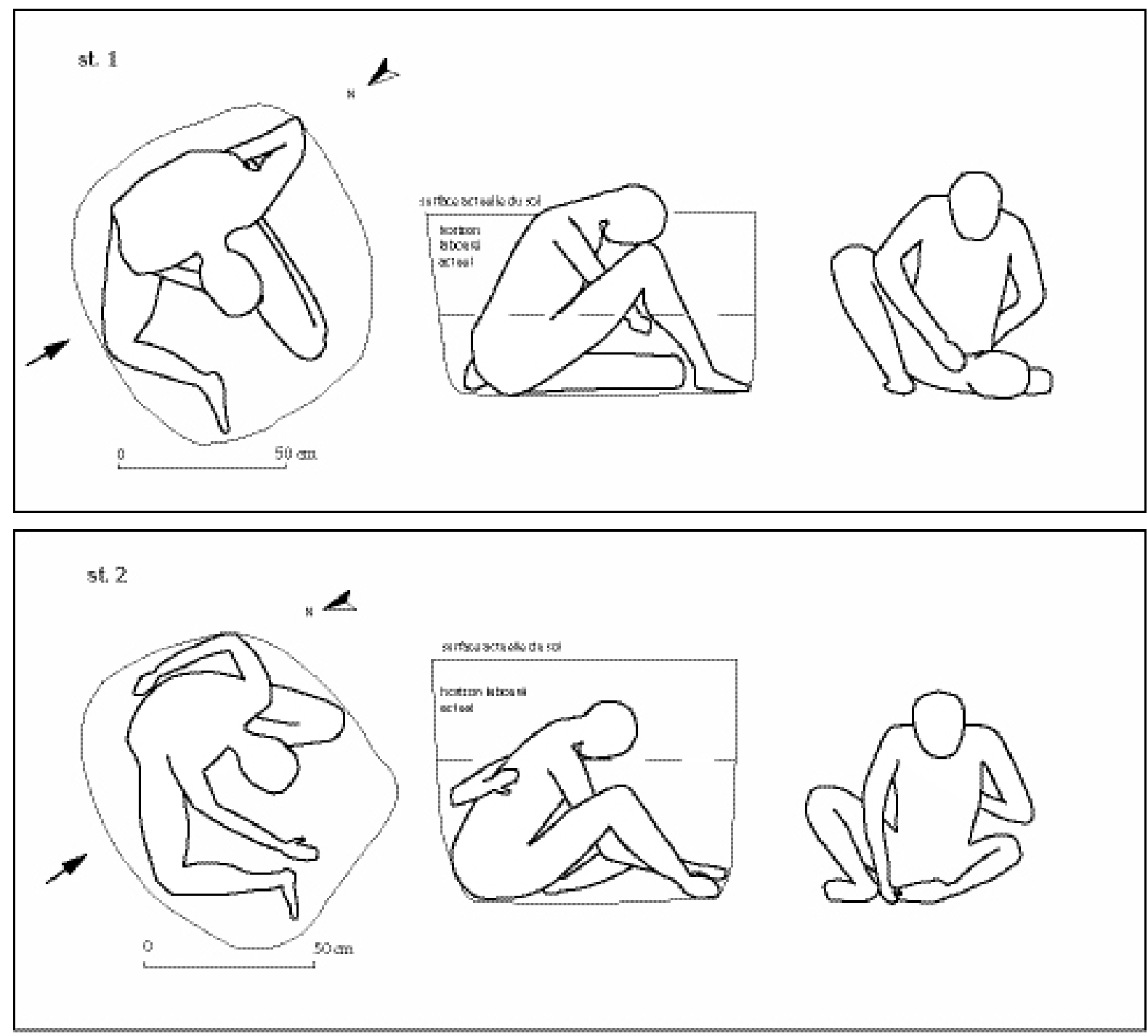

st. 5
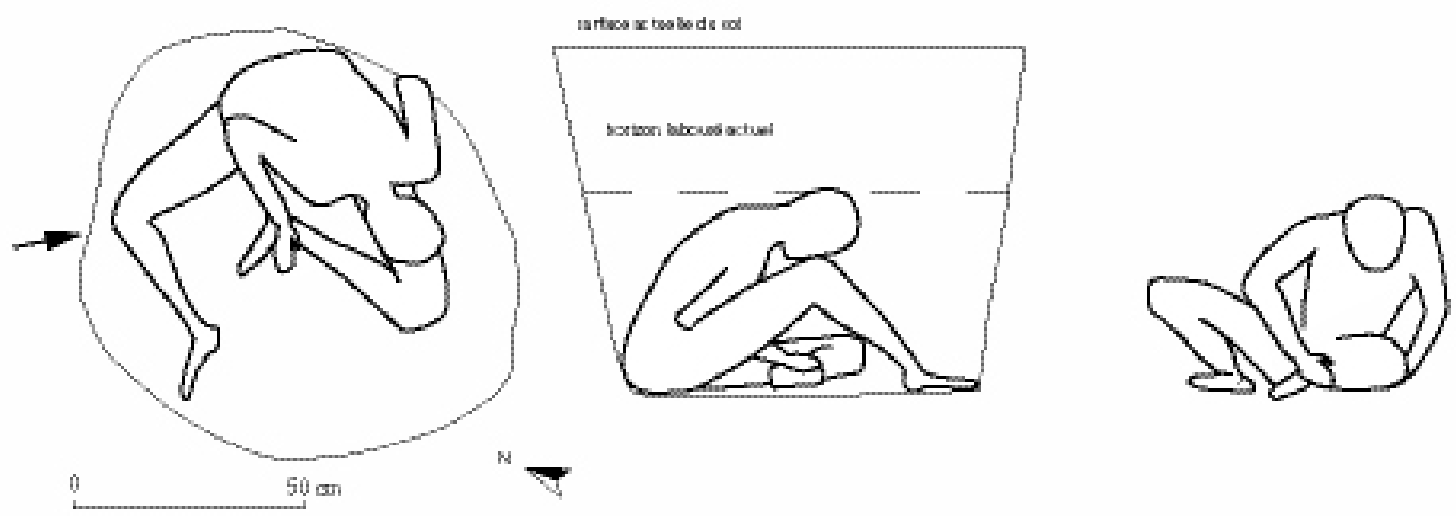

Figure 6 : Reviers, hypothèse de restitution de la disposition intiale des trois corps.

Figure 6: Reconstruction proposal for the initial attitude of the three corpses. 
fosse : quelques dents sont là pour indiquer que le crâne a bien été à un moment donné dans la fosse. Cependant, dans le cas d'un prélèvement après décomposition du corps, on peut supposer que les officiants n'auraient pas également prélevé la partie proximale des scapulas et des humérus : l'absence du crâne et du haut des épaules est plus vraisemblablement due à l'érosion et à la faible profondeur des fosses.

La découverte de ces trois sépultures soulève de nombreuses questions et nous n'avons pas nécessairement les éléments pour y répondre : on peut évoquer notamment la position contrainte de certaines parties du corps et notamment du membre supérieur gauche. C'est particulièrement frappant dans le cas de la st. 2 où il est non seulement derrière le dos, mais en position forcée vu que le poignet est situé $5 \mathrm{~cm}$ plus haut que le coude. Dans les deux autres cas, la flexion est moins importante, mais elle est tout de même contrainte dans le dos pour la st. 1. De même, la position du membre inférieur gauche dans cette dernière sépulture, avec l'intérieur de la cheville vers le fond de la fosse, est fort peu naturelle. On peut supposer l'existence de liens maintenant les membres de ces individus dans des positions inhabituelles. Si le corps était souple au moment de l'inhumation, il faut également imaginer un soutien temporaire le temps du comblement de la fosse pour expliquer que le haut du corps n'ait pas complètement chuté en avant dans le cas de la st. 2; ce n'est pas le cas en revanche pour la st. 5 , puisque le haut du corps avait déjà chuté en avant.

Cette position récurrente a peu de chances de s'expliquer par des raisons opportunistes. Lors de l'inhumation d'un individu adulte, le creusement d'une fosse circulaire d'un mètre de diamètre sur $30 \mathrm{~cm}$ de profondeur peut certes représenter un gain de temps par rapport au creusement d'une fosse quadrangulaire, plus fréquemment rencontrée. Mais une disposition fléchie sur le côté en fotus aurait été plus appropriée pour occuper de manière optimale une fosse circulaire. Enfin, le caractère individuel des trois sépultures ainsi que la position stéréotypée des corps ne plaide pas en faveur d'une inhumation précipitée ou irréfléchie.

Ces positions récurrentes évoquent une mise en scène ritualisée et sont donc certainement porteuses de sens : peutêtre ont-elles un rôle symbolique de punition? On a vu que les positions générales étaient les mêmes (individu assis avec le tronc incliné vers l'avant et vers la gauche, membres fléchis avec effet de contrainte sur le bras gauche), même si, dans le détail, les positions des membres different (en particulier pour ce qui concerne ce même bras gauche) : quel sens doiton apporter à ces différences? Sont-elles significatives ou au contraire anecdotiques?

D'un point de vue biologique, le seul trait commun entre les trois individus dont on puisse être certain est leur âge adulte; seul celui de la st. 5 a pu se voir attribuer un sexe masculin. En l'absence de précisions à la fois sur le sexe et sur la tranche d'âge, on ne peut pas affirmer qu'ils appartiennent à un même groupe social (jeunes adultes masculins par exemple).

D’autres points resteront également sans réponse, comme la fonction de la st. 4, dans le même alignement que les trois fosses sépulcrales, à mi-distance entre les st. 1 et 2 , et celle de la structure 3, plus éloignée. Si l'on intègre au moins la structure 4 au groupe des trois sépultures, alors les distances entre chacune des quatre fosses seraient sensiblement équivalentes : entre 6,70 et $8 \mathrm{~m}$. Les caractéristiques des structures 3 et 4 sont assez proches de celles des trois fosses sépulcrales et on se demande évidemment si elles n'auraient pas pu contenir des corps, ou bien si elles n'ont pas été creusées pour en accueillir. Les dimensions et le remplissage de la st. 3 sont quasiment identiques; en revanche, la st. 4 est nettement plus allongée et son remplissage diffère légèrement.

Au cours de la fouille, trois premières hypothèses avaient été émises pour tenter d'expliquer le sens de ces sépultures inhabituelles : la première était qu'il s'agissait d'une partie marginale de la grande nécropole du Haut Moyen Âge, sur laquelle on observerait un traitement particulier des défunts. Avec la datation radiocarbone, cette première hypothèse peut être rejetée. La seconde hypothèse était celle de fosses sépulcrales anecdotiques, liées à un épisode de conflit ou d'insécurité, générant l'enfouissement sommaire des défunts. Dans ce cas, la fosse circulaire est-elle la plus appropriée? Les exemples les plus récents nous montrent que dans les cas où il faut enterrer rapidement plusieurs individus (conflit, épidémie, etc.), c'est la fosse multiple qui est la plus fréquemment utilisée, comme à Issoudun lors d'un épisode d'épidémie et de famine (Blanchard et al., 2003; Poulle, 2007); ce n'est donc pas ce type de fosse qui serait retenu, mais la position inhabituelle observée à Reviers n'est pas non plus la plus aisée à mettre en place, à moins qu'il ne s'agisse d'une position ante mortem non modifiable. Si cette deuxième hypothèse n'est pas la plus plausible, on ne peut l'écarter complètement, car il n'est pas assuré qu'en période de conflit ou d'épidémie, les individus du Second âge du Fer se comportent comme ceux de l'époque moderne.

La troisième hypothèse évoquée au moment de la fouille a été celle de fosses liées à des pratiques cultuelles ou sacrificielles : en effet, des exemples de traitements inhabituels des corps ont été découverts dans des contextes de sanctuaire. À Fesques en Seine-Maritime (Mantel 1997), l'ensemble cultuel se met en place à partir du $\mathrm{III}^{\mathrm{e}}$ siècle avant J.-C.; parmi les nombreuses structures mises au jour, une série de fosses fouillées à proximité du fossé délimitant la zone sacrée a livré des pieds humains. L'analyse faite sur ces restes démontre une pratique de suspension de corps d'adultes jusqu'à dislocation, le reste des corps étant jeté dans les 
fossés d'enceinte où on les trouve mêlés à des ossements animaux. La position des corps par rapport à la topographie du site y est cependant à l'opposé de celle de Reviers : les individus font face au sanctuaire qui est situé sur le sommet d'une butte. À Gournay-sur-Aronde dans l'Oise (Brunaux et al., 2003), de nombreux os humains ont été découverts en situation résiduelle, après avoir subi de nombreuses manipulations comme le dépeçage et la décollation. Dans ce cas, l'hypothèse sacrificielle est la plus probable, comme dans celui de nombreux autres sites ayant livré des fragments de corps humains, on peut citer entre autres Bailleul-surThérain (Woimant, 1995), Mouvres (Salomon, 1913) ou le sanctuaire bien connu de Ribemont-sur-Ancre (Brunaux, 1999).

Tous ces exemples ont en commun la fragmentation et la manipulation des corps après la mort; on ne peut donc attacher le site de Reviers à ces ensembles en raison de la conservation de l'intégrité du corps dans les trois sépultures. Leur signification sera nécessairement différente de celle accordée à des fragments humains dispersés dans des fosses ou des fossés.

Il faut donc chercher des points de comparaison parmi les sépultures atypiques publiées ayant livré des corps entiers en position assise. Si l'on remonte dans le temps, on peut signaler celles mises au jour à La Saulsotte dans l'Aube (Piette $e t$ al., 2005); elles sont datées du Bronze final. La sépulture détaillée dans la publication est une fosse circulaire de $95 \mathrm{~cm}$ de diamètre, profonde de $20 \mathrm{~cm}$, dans laquelle le corps d'une jeune femme est placé en position assise, avec la tête à l'ouest et les pieds à l'est; les membres inférieurs sont repliés à l'avant et sur le côté gauche, les membres supérieurs sont fléchis. Les auteurs proposent de restituer une décomposition dans une succession d'enveloppes souples, formant un "paquet funéraire ", avec un renforcement du fond par un matériau un peu plus rigide et des liens imposant une rigidité par endroits. Si la position de l'individu et les dimensions de la fosse sont proches de celles de Reviers, l'espace de décomposition en revanche n'est pas le même. Pour la même période, à Barbey en Seine-et-Marne (Rottier, 2005), les individus ont été déposés assis sur leurs talons, dans une position très contractée qui a permis de restituer l'existence d'un coffre; nous sommes donc très éloignés des sépultures de Reviers. À Avenches, dans le canton de Vaud en Suisse, deux squelettes ont été fouillés en 1992 (Moinat, 1993) : ils sont en position assise avec le crâne légèrement en avant du corps, mais présentent une différence dans la position des membres. L'individu de la tombe 1 a les membres inférieurs fléchis vers l'extérieur, les membres supérieurs légèrement fléchis avec les mains en appui sur les cuisses. L'individu de la tombe 2 a le membre inférieur gauche fléchi sur le fond de la fosse, le droit est moins fléchi vers l'extérieur du corps; le membre supérieur gauche est fléchi le long du corps et le droit fléchi avec la main devant le bassin; le crâne a basculé en avant et vers la droite. Les deux individus se sont décomposés en espace colmaté.

À Acy-Romance dans les Ardennes (Lambot et Méniel, 2000), parmi les 16 ha du site, les fouilleurs ont notamment mis en évidence une vaste place communautaire et cultuelle, délimitée par un fossé palissadé et ayant servi entre autres de lieu d'abattage et de découpe d'animaux, ainsi qu'une série de cinq constructions interprétées comme des bâtiments cultuels. Devant l'un de ces bâtiments, de petites fosses circulaires ont livré 19 individus inhumés en position assise, pieds joints et membres inférieurs écartés, la tête reposant sur les pieds. L'hypothèse des auteurs, bien connue aujourd'hui, est que chaque corps était placé dans une caisse de bois et descendu dans un puits qui n'avait pas pu servir à puiser de l'eau en raison de la profondeur de la nappe phréatique. Après être resté durant un temps suffisant pour la dessiccation naturelle des tissus, il était probablement mis à sécher dans un endroit venté puis enterré sur l'esplanade. Ce n'est pas le lieu ici pour revenir sur cette interprétation; on peut toutefois noter que la position des individus est proche de celle observée à Reviers, sans être tout à fait identique. En revanche à Acy-Romance, les dimensions des fosses sont plus petites ( $85 \mathrm{~cm}$ de diamètre en moyenne) et les squelettes n'occupent pas systématiquement tout l'espace disponible.

À Mormont (Canton de Vaud, Suisse), les dernières campagnes de fouille ont mis au jour des fosses recelant plusieurs individus associés à des restes animaux (Dietrich et al., 2009). Ils sont incomplets et ont été l'objet de mutilations (bris de membres, exposition au feu). Les auteurs considèrent qu'on est là hors des contextes funéraires traditionnels et hors des sanctuaires de type guerrier. Si ces considérations s'appliquent à Reviers, la comparaison entre les deux sites s'arrête là, tant les fosses du Mormont sont inhabituelles.

À Reviers, il est difficile de débattre sur un éventuel contexte cultuel du site compte tenu de l'absence apparente de bâtiment cultuel ou de fossé pouvant délimiter un espace sacré. Peut-être existe-t-il à proximité (rappelons que l'espace qui a pu être dégagé au cours de l'opération est restreint) mais, si l'hypothèse d'un site cultuel est possible, elle est invérifiable dans l'immédiat.

En revanche, d'après les exemples présentés ici à titre de comparaison et dans la mesure de ce qui a pu être observé, il paraît difficile d'assurer l'hypothèse sacrificielle pour les individus de Reviers : pas de traitement particulier du crâne, ni décollation, pas de traces de dépeçage sur les ossements ni traumatisme, et l'intégrité du corps semble avoir été conservée.

D'autres sépultures semblables à celles de Reviers ont été mises au jour récemment en divers endroits du territoire 
français. On peut citer notamment le site de Batilly-enGâtinais dans le Loiret (Liégard, 2007) et celui du Champ des Rochers à Soyaux en Charente (Gomez de Soto et Lejars, 2009). Tout récemment, la fouille d'un sanctuaire à SaintJust-en-Chaussée dans l'Oise (Malrain et al., 2008) a permis la mise au jour de huit sépultures à inhumation dont la configuration générale est très proche de celles de Reviers. Une remise en contexte générale permettra certainement de mieux appréhender ces sépultures particulières et de tenter d'expliquer leur place au sein des pratiques funéraires et cultuelles de la fin de l'âge du Fer.

\section{Bibliographie}

Blanchard, P., Castex, D., Souquet-Leroy, I. et Poulle, P., 2003 - Issoudun, Centre de l'image et du multimédia, Archéopages, $\mathrm{n}^{\circ}$ 9, p. 40-41.

BrunauX, J.-L. (dir.), 1999 - Ribemont-sur-Ancre (Somme). Bilan préliminaire et nouvelles hypothèses, Gallia, 56, p. 177283.

Brunaux, J.-L., Malagoli, Cl., Bataille, G. et Lambot, B., 2003 - La France du Nord (dossier «Cultes et sanctuaires en France à l'âge du Fer "), Gallia, 60, p. 9-73.

Desloges, J., Forfait, N., Herard, B. et San Juan, G., 1999 - Les recherches aériennes dans la "Plaine de Caen". Une contribution à l'étude des Âges des Métaux en BasseNormandie (France). Actes du colloque international d'archéologie aérienne (Amiens 1992) - Hommage à Roger Agache, Revue Archéologique de Picardie, 17, p. 417-435.

Dietrich, E., Méniel, P., Moinat, P. et Nitu, C., 2009 - Le site helvète du Mormont (Canton de Vaud, Suisse) : résultats de la campagne de 2008, Bulletin de l'AFEAF, 27, p. 21-25.

Gomez de Soto, J. et Lejars, T. (coord.) ; Bertrand, B., Boulestin, B., Ducongé, S., Kerouanton, I. et Robin, K., 2009 - Les lieux de culte des âges du Fer en Centre-Ouest, in Bertrand, I., Duval, A., Gomez de Soto, J. et Maguer, P. (dir.), Les Gaulois entre Loire et Dordogne (Actes du XXXI colloque de l'AFEAF, Chauvigny 2007, t. I), Chauvigny, Association des Publications Chauvinoises (Mémoire XXXIV), p. 121-132.

Lambot, B. et Meniel, P., 2000 - Le centre communautaire et cultuel du village d'Acy-Romance dans son contexte régional, in Verger, S. (éd.), Rites et espaces en pays celte et méditerranéen. (Actes de la table ronde de Rome, 1997), Rome, Ecole française de Rome (vol. 276), p. 7-139.

LiÉgard, S., 2007 - Le site des Pierrières à Batilly-en-Gâtinais (Loiret), un établissement aristocratique senon traversé par l'autoroute A19, Bulletin de l'AFEAF, 25, p. 51-52.

Malrain, F., Descheyer, N. et Pinard, E., 2008 - Le sanctuaire gaulois et gallo-romain de Saint-Just-en-Chaussée, Rapport d'activités 2008, INRAP., p. 36-39.

Mantel, E. (dir.), 1997 - Le sanctuaire de Fesques " Le Mont du Val aux Moines ", Seine-Maritime, Nord-Ouest Archéologie (vol. 8), $359 \mathrm{p}$.

Moinat, P., 1993 - Deux inhumations en position assise à Avenches, Bulletin de l'Association Pro Aventico, 35, p. 5-12.

Murail, P., BruzeK, J., Houët, F. et Cunha, E., 2005 - DSP: a tool for probabilistic sex diagnosis using worldwide variability in hip-bone measurements, Bulletins et Mémoires de la Société d'Anthropologie de Paris, 17 (n 3-4), p. 167-176.

Olivier, G., 1960 - Pratique anthropologique, Paris, éd. Vigot, $299 \mathrm{p}$.

Piette, J., Rottier, S. et Depierre, G., 2005 - Les pratiques funéraires au début du Bronze final dans les nécropoles de BarbuiseCourtavant et de la La Saulsotte (Aube), in Mordant, D. et Depierre, G. (dir.), Les pratiques funéraires à l'âge du Bronze en France (Actes de la table ronde de Sens-en-Bourgogne, 1998), Paris, éditions du CTHS, p. 433-457.

Poulle, P., 2007 - Le grand cimetière d'Issoudun (Indre) et ses sépultures multiples (fin XviI ${ }^{\mathrm{e}}$-début XVIII ${ }^{\mathrm{e}}$ s.). Premiers résultats de l'étude historique et démographique, in Castex, D. et Cartron, I. (dir.), Épidémies et crises de mortalité du passé (Actes des séminaires - année 2005 - de la Maison des Sciences de l'Homme), Bordeaux, éd. Ausonius (coll. «Études ", 15), p. 77-108.

RotTiER, S., 2005 - Pratiques funéraires originales de la phase ancienne du Bronze final de Barbey "Les Cent Arpents " (Seine-et-Marne), in Mordant, D. et Depierre, G. (dir.), Les pratiques funéraires à l'âge du Bronze en France (Actes de la table ronde de Sens-en-Bourgogne, 1998), Paris, CTHS, p. 459-474.

Salomon, A., 1913 - Le charnier gaulois de Moeuvres, Bulletin de la Société préhistorique française, 10, p. 319-322.

Woimant, G.-P., 1995 - Carte Archéologique de la Gaule, l'Oise, Paris, Maison des Sciences de l'Homme, 570 p. 
